\title{
Affordable Activity Monitors Can Improve Post-Surgical Care in Bariatric Surgery Patients
}

\section{Ryan ER Reid and Ross E Andersen*}

Department of Physical Education and Kinesiology, McGill University, Montreal, Quebec, Canada

*Corresponding author: Ross E Andersen, Department of Physical Education and Kinesiology, McGill University, Montreal, Quebec, Canada, Tel: 5143984184; Fax: 5143984186; E-mail: ross.andersen@mcgill.ca

Received date: June 22, 2014, Accepted date: December 26, 2014, 2014Published date: January 1, 2015

Copyright: (C) 2015 Andersen RE et al. This is an open-access article distributed under the terms of the Creative Commons Attribution License, which permits unrestricted use, distribution, and reproduction in any medium, provided the original author and source are credited.

\begin{abstract}
Bariatric surgery is an effective method of dramatically reducing weight and minimizing the appearance of comorbidities in several obese individuals. Regardless of weight lost post-surgically, some patients fail to increase their amount of physical activity and remain overly sedentary. Furthermore, in the years following surgery, certain patients re-gain some of the weight that they had initially lost. A proposed explanation for this lack of long-term weight loss maintenance is the lack of attendance to long-term post-surgical follow-up visits. If health care providers are unable to monitor the progress of their patients post-surgically, it is impossible for them to provide new strategies that will promote better long-term weight loss success. Recently, new and more affordable activity monitoring devices have become accessible with the ability to log information about physical activity and nutrition wirelessly to web based software. This software enables the information to be accessed by anyone with internet access and the correct password. These devices give the opportunity for patients to communicate with their health care providers without the need for in person follow-up visits and offer an affordable option for better and more reliable long-term follow-up care after bariatric surgery.
\end{abstract}

Keywords: Bariatric Surgery; Sedentary Behavior; Physical Activity; Long-Term Follow-Up

\section{Commentary}

The prevalence of obesity in the United States of America has risen from $30.2 \%$ in the year $2000[1,2]$ to $35.7 \%$ in $2010[1,2]$ and the prevalence of class III obesity (BMI $\geq 40 \mathrm{Kg} / \mathrm{m} 2$ ) was reported to be $6.3 \%$ in 2009-2010 [1]. In Canada, the prevalence of individuals categorized as class III obese has risen steadily from $0.4 \%$ in 1990 [3] to $2.8 \%$ of the population in 2008 [4]. Obesity is associated with several other chronic diseases including: coronary artery disease, type II diabetes, certain cancers [5], sleep apnea [6], anxiety and depression [7]. The rising prevalence of class III obesity combined with obesity's association with the previously mentioned co-morbidities makes this a growing public health concern.

Currently, bariatric surgery is the preferred treatment option for severely obese individuals, especially those with any one or combination of the many obesity related co-morbidities [8]. In 2006, there were a total of 112,999 bariatric procedures performed in the United States [9], and in Canada, there was a $61 \%$ increase in bariatric procedures from 2004 (1152 surgeries) to 2008 (1881 surgeries) [10]. The growing popularity of bariatric surgery is partially due to the fact that it is known to produce not only initial short-term weight loss and reduction in co-morbidities but also long-term weight loss maintenance years after surgery [11].

There are three basic categories of bariatric surgery: 1. Restrictive procedures in which the size of the stomach is reduced to minimize the amount of nourishment that can be ingested at any one time, 2 Malabsorptive procedures where a section of the small intestine is removed to reduce the body's ability to absorb some of the nutrients from the food that is ingested, and 3. Combined procedures that use aspects of both restrictive and malabsorptive procedures [12]. These techniques all yield highly effective immediate post-surgical weight loss and reduction in the appearance of co-morbidities such as type 2 diabetes [13]. Although bariatric surgery is very effective, each type of procedure has its own short- and long-term side effects that need to be attended to by the health care provider at post-surgical follow-up visits. However, regardless of the type of surgery, long-term postsurgery patients typically regain some of the weight that was initially lost [14].

Physical activity has been shown repeatedly to be a cornerstone of weight loss management programs and is a good predictor of longterm weight maintenance among individuals who have lost significant amounts of weight [15]. Furthermore, higher levels of physical activity are associated with reduced risk of heart disease, cancer [16] and better mental health among bariatric surgery candidates [7].

It has been shown that bariatric surgery candidates spend $80 \%$ of their waking hours in sedentary behaviors, [17] which is $20-30 \%$ more time than those in the rest of population [18]. Higher levels of sedentary behavior is an independent risk factor for weight gain, obesity [19] as well as poorer metabolic profiles independent of physical activity [20] and should be considered alongside physical activity when discussing the long-term post-surgical lifestyle recommendations for these individuals.

It has recently been shown that there is no change in the number of steps/day or the amount of daily sitting time from pre- to 3 and even 6 months following bariatric surgery. Moreover, using cross sectional data from a group of individuals who had bariatric surgery on average 8.88 years ago demonstrated that their number of steps/day and hours of daily sitting time still remained unchanged compared to the previously mentioned cohort. All of which were classified into a low active category based on Tudor-Locke's steps/day classification system 
[21]. At the risk of weight re-gain and increased risk of co-morbidities re-appearing, something needs to be done either pre- or immediately post-surgery to address this lack of change in physical activity and sedentary habits.

Recently, affordable, attractive looking and small sized personal activity monitors have become available to the general public. These monitors, aside from personal activity monitoring, offer a unique feature of wireless linking of the monitored information to a website that can be accessed through any computer or mobile device with internet access by whoever possess the password. This feature affords primary health care professionals the ability to track the activity of their patients pre- or post-surgery in real time. Companies such as Fitbit $^{\mathrm{mm}}$ (San Francisco, CA), Jawbone ${ }^{\mathrm{mx}}$ (San Francisco, CA) and MOVable ${ }^{\mathrm{sw}}$ (Brecksville, $\left.\mathrm{OH}\right)$ offer these wearable accelerometers costing from $\$ 25$ to $\$ 150$ and have abilities ranging from recording steps/day and distance walked to calculating the minutes of and intensity of the activity recorded throughout the day.

All of these devices measure steps/day, miles walked, minutes of vigorous activity throughout the day, amount of sleep, as well as nighttime disturbances and can help to track calories consumed and burned. Many of these devices have free applications for smartphones that make self-monitoring of all of these variables easier, allowing patients to makes notes, sync their devices and log food as they wish throughout the day, rather than logging into the website when they are next near a computer. This feature helps to ensure reliable real time information logging and helps to remove some of the error that comes with missing or faulty logging later on in the day after the event has taken place. A further benefit from using this software is that caloric and activity related goals can be set before, during or after monitoring has begun. Set effectively with the help of a kinesiologist and/or nutritionist, the device can notify the patient if they have met their daily goals and even prompt the wearer with positive reinforcing messages and reminders to meet the goals set to their profile on the device. These prompts along with the ability link multiple groups of patients together over the internet could be a terrific motivator for some patients to be more active while removing the inaccuracy of selfreported information from the overall experience. Study of this population has shown that bariatric surgery patients perceive themselves as more active than they actually are when compared to objective accelerometry [22]. The objective data generated by these devices is important for healthcare professionals to have in order to prescribe an appropriate increase in physical activity tailored specifically for each patient.

At the end of each week a summary detailing the daily and weekly average of all of the variables monitored throughout the week can be automatically sent to the primary health care provider and the patient alike. The health care provider can then examine the activity patterns of the patient, go over it with them and promote healthier options where sitting time can be reduced and the amount as well as intensity of activity can be improved. The main advantage of using these more sophisticated devices over simpler activity monitors like a pedometer is that the data is automatically saved online for later review. Therefore, the patient could wear the device for one week leading up to a post-surgical follow-up visit and the data can be accessed, at any time before, during or even after the visit depending on the preference of the health care provider and patient.

A key problem with the long term care of bariatric surgery patients is the inability to maintain good adherence to long term follow up visits. These devices can help to improve the information exchange that is required during long-term post-surgical follow up visits regardless of whether the patient comes to the clinic or not. This technology enables health care professionals to have access to more objective information concerning the patient's activity and nutritional habits without meeting with the patient face to face. Therefore, if the attending physician is unable to meet with the patient concerning their level of physical activity, the recording can be given to a part-time or off-cite kinesiologist who can review the activity data for the physician and design an appropriate exercise routine and some helpful suggestions to accompany detailed instructions to the patient. This type of document can be prepared ahead of time and given to the patient by the physician at the time of follow-up, or even e-mailed to the patient as a PDF document, further eliminating the need for the patient to visit the hospital to acquire the important counseling. Nutritionists can have access to documented food logs and estimated number of calories burned through use of the devices' websites, allowing them to prepare recommended foods and meal plans for patients before meeting with them at post-surgical visits, or to e-mail to them saving a trip to the hospital.

New research shows that patients awaiting bariatric surgery are insufficiently active and that this level of inactivity does not change in the months following surgery despite dramatic weight loss [23]. Other longitudinal work has shown that on average 10 years after surgery patients demonstrate almost identical levels of inactivity but now with a percentage of weight regained [24]. Being that physical activity is a cornerstone of any weight loss maintenance regime [15] these devices allow healthcare professionals to more accurately monitor, counsel, and prescribe physical activity to their patients in order to avoid this weight regain that is seen long-term after the surgery

Most clinics and hospitals that conduct bariatric surgeries offer some type of pre- and post-surgical support groups. This is where groups of patients meet to discuss their experiences with the surgery, to gain information and new perspectives from other individuals who are going through a similar experience. The web based software for these activity monitoring devices allows for groups of people to form communities in which multiple people can have access and view others' online activity and food logs. Groups of patients can use these devices in support groups to have activity competitions, improve adherence to physical activity guidelines and promote a physically active environment.

There are some minor barriers on recommending use of these devices. The key problem is that the patients must have access to them. Although $\$ 100$ is much less expensive than more research focused activity monitors, it may still prevent patients from purchasing them. Secondly, in order to use these devices to their full potential, patients will need access to a computer with internet, or ideally a smartphone. Although not required for the basic activity monitoring function of the devices, a smartphone allows patients to log in real time and have immediate feedback concerning their tracked values. Finally, the patients using these devices must be somewhat technologically savvy. Although most adults 50 years of age and under would find these devices fairly simple to operate, more aged individuals may find them difficult and confusing to use.

If these more expensive and complex devices prove to be too expensive or technologically advanced, even extremely simple pedometers costing under $\$ 10$ with digital displays can be worn and used to monitor activity effectively. Patients can record the number of steps/day for a given period of time on a calendar or sheet of paper. This activity log can be brought to the patients follow up visits post- 
surgically or e-mailed to the health care professional and be used in a similar manner as the web based recordings. Although this option may be considered slightly less objective, it would still represent a low cost viable option to increase patients' awareness of their level of physical activity and allow the health care provider to further counsel the patient on some physically active options for their daily life based on the results obtained.

In conclusion, these new more affordable activity monitoring devices combined with their novel web based softwares seem to offer a unique and relatively easy way for health care providers to track and enhance their patients' level of daily activity. Adoption of this technology in bariatric clinics pre- or post-surgery may help to reduce some of the excess sitting time and high levels of inactivity that are often common in this very unique population. It is hoped that these devices can help promote better long-term weight control and healthier living.

\section{Acknowledgement}

Data Collected Through Funds Provided by Canadian Institutes of Health Research.

\section{References}

1. Ogden CL, Carroll MD, Kit BK, Flegal KM (2012) Prevalence of obesity in the United States, 2009-2010. Centers for Disease Control and Prevention National Center for Health Statistics 82:1-8.

2. Sturm R (2007) Increases in morbid obesity in the USA: 2000-2005. Public Health. 121:492-6.

3. Katzmarzyk PT, Mason C (2006) Prevalence of class I, II and III obesity in Canada. CMAJ 174: 156-157.

4. Canada GO, editor. Statcan [Internet]. [cited 2013 Aug 13].

5. Calle EE, Rodriguez C, Walker-Thurmond K, Thun MJ (2003) Overweight, obesity, and mortality from cancer in a prospectively studied cohort of U.S. adults. N Engl J Med 348: 1625-1638.

6. Charuzi I, Lavie P, Peiser J, Peled R (1992) Bariatric surgery in morbidly obese sleep-apnea patients: short- and long-term follow-up. Am J Clin Nutr 55: 594S-596S.

7. King WC, Kalarchian MA, Steffen KJ, Wolfe BM, Elder KA, Mitchell JE (2013) Associations between physical activity and mental health among bariatric surgical candidates. Journal of Psychosomatic Research. 74:1619.

8. O'Brien PE (2010) Bariatric surgery: mechanisms, indications and outcomes. J Gastroenterol Hepatol 25: 1358-1365.
9. Livingston EH (2010) The incidence of bariatric surgery has plateaued in the U.S. Am J Surg 200: 378-385.

10. Christou NV (2011) Access to bariatric (metabolic) surgery in Canada. Canadian Journal of Diabetes 35:123-8.

11. Sjöström L, Lindroos AK, Peltonen M, Torgerson J, Bouchard C, et al. (2004) Lifestyle, diabetes, and cardiovascular risk factors 10 years after bariatric surgery. N Engl J Med 351: 2683-2693.

12. Kissane NA, Pratt JS (2011) Medical and surgical treatment of obesity. Best Pract Res Clin Anaesthesiol 25: 11-25.

13. Mingrone G, Panunzi S, De Gaetano A, Guidone C, Iaconelli A, et al. (2012) Bariatric surgery versus conventional medical therapy for type 2 diabetes. N Engl J Med 366: 1577-1585.

14. Mathus-Vliegen EM (2007) Long-term health and psychosocial outcomes from surgically induced weight loss: results obtained in patients not attending protocolled follow-up visits. Int J Obes (Lond) 31: 299-307.

15. Andersen RE, Wadden TA, Bartlett SJ, Zemel B, Verde TJ, et al. (1999) Effects of lifestyle activity vs structured aerobic exercise in obese women: a randomized trial. JAMA 281:335-340.

16. Blair SN, Kohl HW 3rd, Paffenbarger RS Jr, Clark DG, Cooper KH, et al. (1989) Physical fitness and all-cause mortality. A prospective study of healthy men and women. JAMA 262: 2395-2401.

17. Bond DS, Unick JL, Jakicic JM, Vithiananthan S, Pohl D, et al. (2011) Objective assessment of time spent being sedentary in bariatric surgery candidates. Obes Surg 21: 811-814.

18. Matthews CE, Chen KY, Freedson PS, Buchowski MS, Beech BM, et al. (2008) Amount of time spent in sedentary behaviors in the United States, 2003-2004. Am J Epidemiol 167: 875-881.

19. Hu FB, Li TY, Colditz GA, Willett WC, Manson JE (2003) Television watching and other sedentary behaviors in relation to risk of obesity and type 2 diabetes mellitus in women. JAMA 289: 1785-1791.

20. Owen N, Healy GN, Matthews CE, Dunstan DW (2010) Too much sitting: the population health science of sedentary behavior. Exerc Sport Sci Rev 38: 105-113.

21. Tudor-Locke C, Bassett DR Jr (2004) How many steps/day are enough? Preliminary pedometer indices for public health. Sports Med 34: 1-8.

22. Andersen KM, Carver TE, Reid RER, Bartlett SJ, Court O, at al. (2014) Self-Reported and Objectively Measured Physical Activity and Sedentary Behavior in People Who Have Had Bariatric Surgery. Medicine \& Science in Sports \& Exercise 46(5):496-497.

23. Babineau O, Carver TE, Christou NV, Andersen RE (2012) Objectively Quantifying Physical Activity And Sedentary Behavior 10?Years Following Bariatric Surgery. In: Vol 44. San Francisco 267-269.

24. Reid RER, Carver TE, Reid TG, Court O, Andersen RE (2014) The Effect of Sleep Time on Day Sedentary Time in Long-Term Post-Bariatric Surgery Patients. Medicine \& Science in Sports \& Exercise 46(5):796. 